\title{
Systematic study of the de-excitation of neutron- rich nuclei produced in different fission reactions
}

\author{
Andreas Oberstedt ${ }^{1,2, *}$, Angélique Gatera ${ }^{3}$, Alf Göök ${ }^{3}$, Matthieu Lebois $^{4}$, \\ Stephan Oberstedt ${ }^{3}$, Liqiang $\mathrm{Qi}^{4}$, Christelle $\mathrm{Schmitt}^{5}$, and Jonathan N. Wilson ${ }^{4}$ \\ ${ }^{1}$ Extreme Light Infrastructure - Nuclear Physics (ELI-NP) / Horia Hulubei National Institute for Physics \\ and Nuclear Engineering (IFIN-HH), 077125 Bucharest-Magurele, Romania \\ ${ }^{2}$ Department of Physics and Astronomy, Uppsala University, 75120 Uppsala, Sweden \\ ${ }^{3}$ European Commission, DG Joint Research Centre, Directorate G - Nuclear Safety and Security, Unit \\ G.2 Standards for Nuclear Safety, Security and Safeguards, 2440 Geel, Belgium \\ ${ }^{4}$ Université Paris-Saclay, CNRS/IN2P3, IJCLab, 91406 Orsay, France \\ ${ }^{5}$ Institut Pluridisciplinaire Hubert Curien, 23 rue du Loess, B.P. 28, 67037 Strasbourg Cedex 2, France
}

\begin{abstract}
In this paper we present results from two recent studies, both related to the emission of prompt fission $\gamma$ rays. Firstly, we have analyzed data from the reaction ${ }^{235} \mathrm{U}(\mathrm{n}, \mathrm{f})$ induced by fast neutrons of average energy $\bar{E}_{n}=1.7 \mathrm{MeV}$. The deduced spectral characteristics are an average multiplicity $\bar{M}_{\gamma}=7.11 \pm$ $0.44 \gamma$ rays per fission and an average total $\gamma$-ray energy release in fission $\bar{E}_{\gamma, \text { tot }}$ $=5.51 \pm 0.46 \mathrm{MeV}$, corresponding to an average $\gamma$-ray energy $\bar{\epsilon}_{\gamma}=0.77 \pm$ $0.08 \mathrm{MeV}$. Secondly, we have addressed - and answered - the question how those characteristics in general depend on the width of the chosen prompt time window and the timing resolution, determined by the employed detectors and electronics. The conclusion is that once this is known, it is possible to compare results from different experiments in a more meaningful way.
\end{abstract}

\section{Introduction}

The energy released in nuclear fission is distributed in kinetic and excitation energy of the two fragments. The excitation energy manifests itself in fragment deformation and intrinsic excitation energy. The first step of fission fragment de-excitation takes place within a few nanoseconds after scission through the successive emission of prompt neutrons. Then, in a simplified picture, when the fragments' remaining excitation energy is lower than the neutron binding energy, $\gamma$ rays are emitted. Thereafter, de-excitation continues through $\beta$-decay towards isotopes in the valley of stability and subsequent (delayed) neutron and $\gamma$-ray emission $[1,2]$. The prompt neutrons are important for maintaining a chain reaction in a reactor, while delayed neutrons allow reactor control. Prompt fission $\gamma$-ray emission contributes to the prompt heat released in fission, whereas the delayed $\gamma$-ray emission is counted as decay heat. Hence, the properties of the emitted particles prior to the onset of weak decays are important for both nuclear applications as well as for the better understanding of the mechanism of fission fragment de-excitation. While prompt fission neutron emission is being studied

\footnotetext{
*e-mail: andreas.oberstedt@eli-np.ro
} 
during the last 50 years, prompt $\gamma$-ray emission is one of the least understood parts of the fission process and was investigated essentially in the early 1970s and then again since about a decade ago, and only for a limited number of fissioning isotopes (cf. Ref. [3] and references therein).

Concerning the latter, we have contributed in the past years with a number of precise measurements of prompt fission $\gamma$-ray spectra (PFGS) from various systems, i.e. the spontaneous fission of ${ }^{252} \mathrm{Cf}[4,5]$ and ${ }^{240,242} \mathrm{Pu}$ [6], thermal-neutron induced fission on ${ }^{235} \mathrm{U}$ [7] and ${ }^{239,241} \mathrm{Pu}[8,9]$, as well as fast-neutron induced fission on ${ }^{235,238} \mathrm{U}$ [10-12], and quite recently, ${ }^{239} \mathrm{Pu}$ [13]. For those we determined average characteristics like multiplicity, mean energy per photon and total $\gamma$-ray energy released in fission. The obtained results were investigated for their dependences of mass and atomic numbers of the fissioning system as well as the dissipated excitation energy. The purpose of this endeavor was to find a description that allows predicting prompt $\gamma$-ray spectra characteristics for cases that cannot be studied experimentally [14]. In this conference paper, however, we will present new results for PFGS characteristics from ${ }^{235} \mathrm{U}(\mathrm{n}, \mathrm{f})$ and address the question how those characteristics in general depend on the width of the chosen time window and the timing resolution of the employed detectors and electronics. Finally, we will give an outlook on our future activities in this field.

\section{Picking up the threads}

In the very first experiment performed at the directional fast-neutron source LICORNE of IPN Orsay [15] some years ago, prompt-fission $\gamma$ rays were measured with 14 hexagonal $\mathrm{BaF}_{2}$ and three coaxial $\mathrm{LaBr}_{3}: \mathrm{Ce}$ scintillation detectors. The $\gamma$ rays were produced in fission reactions induced by neutrons of average energy $\bar{E}_{n}=1.7 \mathrm{MeV}$ hitting ${ }^{235} \mathrm{U}$ and ${ }^{238} \mathrm{U}$ samples of $10 \mathrm{mg}$ approximate mass each, placed back-to-back at the central cathode position inside a cylindrical twin Frisch-grid ionization chamber. Until quite recently, only $\gamma$ rays detected with the $\mathrm{BaF}_{2}$ scintillators had been analyzed and first results were already published [10]. As stated there, the low counting statistics did not allow to treat the $\mathrm{LaBr}_{3}$ :Ce spectra back then due to difficulties in unfolding the detector responses. Concerning the $\mathrm{BaF}_{2}$ spectra, the thresholds were rather high, approximately $500 \mathrm{keV}$. Moreover, during this experiment we encountered problems associated with dead time, which might have resulted in uncertainties related to a proper normalization of the $\gamma$-ray spectra with respect to the total number of occurred fission events. For the reasons mentioned above, no absolute values for PFGS characteristics (i.e. average multiplicity, average and total $\gamma$-ray energy) could have been deduced. Instead, only ratios for ${ }^{235} U$ and ${ }^{238} U$ spectral properties were given [10]. However, subsequent experiments have provided us now with the means for "picking up the thread" from this previous measurement and analyzing also the spectra taken with the $\mathrm{LaBr}_{3}: \mathrm{C}$ detectors.

One of the prerequisites mentioned above is the recent measurement of prompt $\gamma$ rays from the reaction ${ }^{238} \mathrm{U}(\mathrm{n}, \mathrm{f})$ at $\bar{E}_{n}=1.9 \mathrm{MeV}$ [11]. Taking into account that the average prompt-fission $\gamma$-ray multiplicities for this reaction agree well within $1 \%$ for incident neutrons of 1.7 and $1.9 \mathrm{MeV}$ kinetic energy, which may be inferred from the predictions in Ref. [14], the spectrum at $1.9 \mathrm{MeV}$ can be used to obtain the correct normalization for the spectrum at $1.7 \mathrm{MeV}$ by adjusting the latter to the first one. This was done for the region $0.5 \mathrm{MeV}<$ $E_{\gamma}<6 \mathrm{MeV}$ to reproduce the same multiplicity, and the result is shown in Fig. 1. Since both $\mathrm{BaF}_{2}$ spectra for $\mathrm{n}+{ }^{235} \mathrm{U}$ and $\mathrm{n}+{ }^{238} \mathrm{U}$, respectively, were detected simultaneously, the scaling factor obtained above for $n+{ }^{238} \mathrm{U}$ may be applied to the PFGS for $n+{ }^{235} \mathrm{U}$. This in turn allows also to fix the absolute height of the ${ }^{235} \mathrm{U}(\mathrm{n}, \mathrm{f})$ PFGS taken with the $\mathrm{LaBr}_{3}: \mathrm{Ce}$ detectors to the one observed with the $\mathrm{BaF}_{2}$ detectors.

As mentioned above, counting statistics was rather low for the $\mathrm{LaBr}_{3}: \mathrm{Ce}$ detectors, in contrast to the spectra obtained with the massive $\mathrm{BaF}_{2}$ clusters. For that reason a reliable 


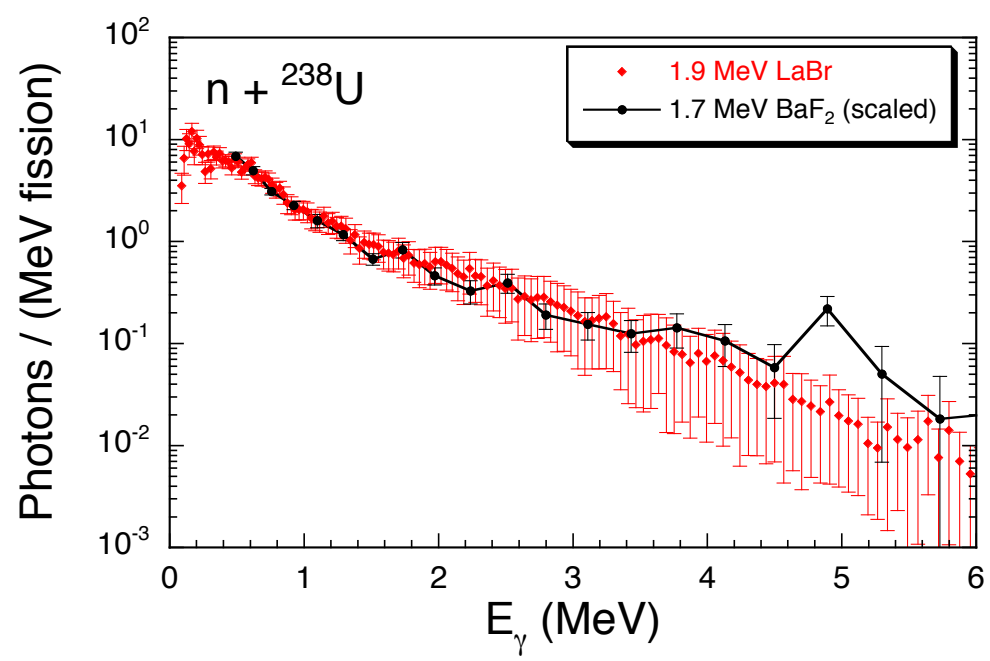

Figure 1. Comparison of the unfolded prompt-fission $\gamma$-ray spectra for $\mathrm{n}+{ }^{238} \mathrm{U}$ at $\bar{E}_{n}=1.9 \mathrm{MeV}$ [11] and $1.7 \mathrm{MeV}$ [10], where the latter was adjusted to the first one (see text for details).

unfolding of the detector response was not possible for the recorded $\mathrm{LaBr}_{3}$ :Ce spectra. However, we observed that the spectra measured in coincidence with the fission of $n+{ }^{235} \mathrm{U}$ and $n+{ }^{238} \mathrm{U}$ exhibit a very similar shape (details will be given elsewhere). Hence, instead of unfolding the measured ${ }^{235} \mathrm{U}(\mathrm{n}, \mathrm{f}) \mathrm{PFGS}$, we apply a transformation function, a procedure that was explained and applied previously to ${ }^{240,242} \mathrm{Pu}$ (sf) PFGS in Ref. [6]. In this case, the transformation function is obtained for the system $n+{ }^{238} \mathrm{U}$, by using the measured $\mathrm{LaBr}_{3}: \mathrm{Ce}$ spectrum at $\bar{E}_{n}=1.7 \mathrm{MeV}$ and the previously determined emission spectrum at $\bar{E}_{n}=$ $1.9 \mathrm{MeV}$. Here we apply the same argument as above that the emission spectra at 1.7 and $1.9 \mathrm{MeV}$ are practically identical, which is corroborated by Fig. 1, at least for $\gamma$ energies below $3 \mathrm{MeV}$. However, this does not imply any restriction, because the low statistics for the $1.7 \mathrm{MeV}$ measurement does not allow to consider $\mathrm{LaBr}_{3}$ :Ce spectra above that energy anyway. As a result, we obtain emission spectra for $n+{ }^{235} U$, as shown in Fig. 2, one from the $\mathrm{BaF}_{2}$ clusters and the other from the $\mathrm{LaBr}_{3}$ :Ce detectors. The $\mathrm{BaF}_{2}$ spectrum here has been scaled according to the $\mathrm{BaF}_{2}$ spectrum from the ${ }^{238} \mathrm{U}(\mathrm{n}, \mathrm{f})$ measurement (cf. Fig. 1), hence both have the same height relative to each other just like it is shown in Ref. [10]. The $\mathrm{LaBr}_{3}: \mathrm{Ce}$ spectrum was adjusted to the $\mathrm{BaF}_{2}$ spectrum by requiring the same integral, i.e. multiplicity, within the overlap region $0.5 \mathrm{MeV}<E_{\gamma}<2.0 \mathrm{MeV}$. Finally, from the combined spectrum, PFGS characteristics for the fast-neutron induced fission of ${ }^{235} \mathrm{U}$ were deduced like described e.g. in Ref. [11]. The results are given in Table 1, together with corresponding values from another recent experiment on the same fissioning system, but induced by thermal neutrons [16]. The spectrum obtained from the latter measurement is also depicted in Fig. 2 for comparison. Apparently, all three spectra agree rather well, as the numbers do in Table 1 - within overlapping uncertainties. For the fast-neutron induced PFGS results, the uncertainties include statistical ones as well as those from the adjustments made and the transformation function. The results will be discussed below.

\section{Comparing apples and oranges}

The coincident measurement of photons and fission fragments gives rise to events of different physical origin [17], of which here only prompt fission $\gamma$ rays are of interest. These events are 


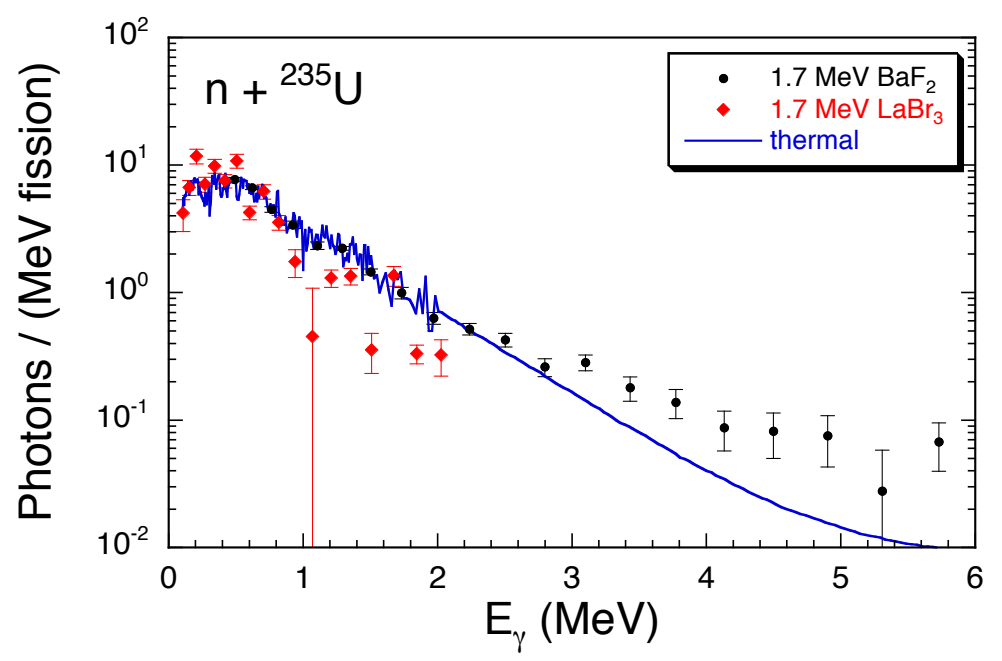

Figure 2. Prompt-fission $\gamma$-ray emission spectra for the reaction $\mathrm{n}+{ }^{235} \mathrm{U}$ at $\bar{E}_{n}=1.7 \mathrm{MeV}$ obtained with the $\mathrm{BaF}_{2}$ clusters [10] and with the $\mathrm{LaBr}_{3}$ :Ce detectors (this work). For comparison a recently measured spectrum from thermal neutrons on ${ }^{235} \mathrm{U}[16]$ is shown too.

easily distinguished by time-of-flight (TOF) measurements and identified as peak in a corresponding TOF distribution. Selecting $\gamma$ rays inside this peak ensures that neutron-induced events are excluded, whereas any background is strongly suppressed by the coincidence condition between photons and fission fragments. During the last 60 years of experimental studies of prompt fission $\gamma$-ray emission, different detector systems, electronics, measurement and data analysis techniques were employed, which may cause difficulties when comparing the obtained results in terms of prompt fission $\gamma$-ray spectra (PFGS) characteristics. Hence, comparing experimental results is sometimes like "comparing apples and oranges". In previous works, the influence of the high energy range (i.e. above $6-10 \mathrm{MeV}$ ) of the detected PFGS [7] as well as low-energy photons (below $500 \mathrm{keV}$ ) [5] has been assessed already.

In order to illustrate the impact of the width of the timing window on PFGS characteristics, we refer here to previously unpublished results from a measurement on ${ }^{252} \mathrm{Cf}(\mathrm{sf})$. In this case both experimental setup and data treatment correspond to what has been described in Ref. [4]. However, for this study different TOF windows were used to select prompt fission $\gamma$ rays and the resulting measured spectra with the corresponding events were unfolded with the response function of the used $58 \mathrm{~mm} \times 58 \mathrm{~mm}$ cylindrical $\mathrm{LaBr}_{3}:$ Ce scintillation detector (for details about the unfolding procedure we refer to Ref. [4]).

Figure 3 shows the measured TAC (= time-to-analogue converter) spectrum for $\gamma$ rays detected in coincidence with fragments from the spontaneous fission of ${ }^{252} \mathrm{Cf}$ as full-drawn line, centered around zero, i.e. the instant of fission. The dotted regions correspond to the

Table 1. Summary of PFGS characteristics from the reaction ${ }^{235} \mathrm{U}(\mathrm{n}, \mathrm{f})$ at different incident neutron energies for the range $0.1 \mathrm{MeV}<E_{\gamma}<6.0 \mathrm{MeV}$.

\begin{tabular}{lccccc}
\hline \hline Detector & $\bar{E}_{n}(\mathrm{MeV})$ & $\bar{M}_{\gamma}$ & $\bar{E}_{\gamma, \text { tot }}(\mathrm{MeV})$ & $\bar{\epsilon}_{\gamma}(\mathrm{MeV})$ & Reference \\
\hline $\mathrm{LaBr}_{3}: \mathrm{Ce} / \mathrm{BaF}_{2}$ & 1.7 & $7.11 \pm 0.44$ & $5.51 \pm 0.46$ & $0.77 \pm 0.08$ & this work \\
$\mathrm{LaBr}_{3}: \mathrm{Ce}$ & thermal & $7.38 \pm 0.18$ & $6.37 \pm 0.20$ & $0.86 \pm 0.03$ & {$[16]$} \\
\hline \hline
\end{tabular}




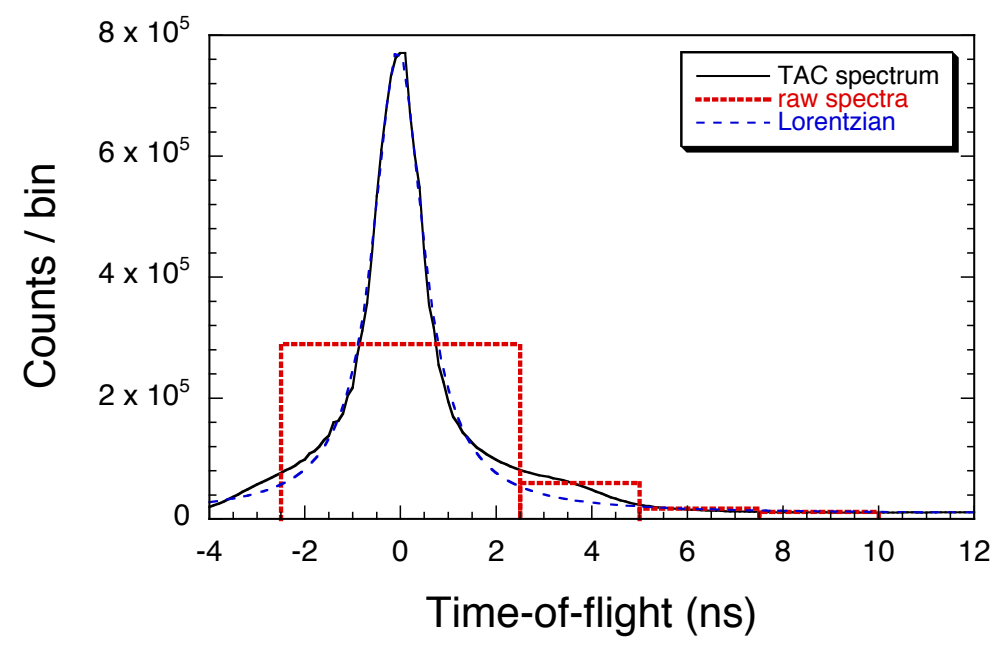

Figure 3. Time-of-flight distribution (denoted by TAC spectrum) of $\gamma$ rays from a coincidence measurement with fission fragments from ${ }^{252} \mathrm{Cf}(\mathrm{sf})$, together with the sum of counts from the corresponding raw spectra averaged over the respective time regions. The Lorentzian is the result of a fit to the measured TAC spectrum.

number of $\gamma$ rays in the measured raw spectra for each of the chosen time-of-flight regions, distributed over the respective bin. The limits are $-2.5,2.5,5,7.5$ and $10 \mathrm{~ns}$, respectively. Background contributions are very small and not depicted. Integrating over the regions separated by the dotted lines and over the TAC spectra within the same boundaries gives the same results. In addition, a Lorentzian is plotted as the fit result to the measured TAC spectrum; this approximation will be used later to parametrize the prompt $\gamma$-ray timing peak.

From the different prompt fission $\gamma$-ray emission spectra and according to the time limits depicted above, average multiplicities, mean energies per photon and average total $\gamma$-ray energies released in fission were then extracted. The obtained results are depicted as full dots in Fig. 4 as function of the upper time limit of the different timing windows, while the lower limit in all cases was $-2.5 \mathrm{~ns}$. The left-hand side shows average prompt fission $\gamma$-ray multiplicities $\bar{M}_{\gamma}$, while the average $\gamma$-ray energies $\bar{\epsilon}_{\gamma}$ are shown to the right. The error bars contain both statistical and systematic uncertainties. It is obvious that the average multiplicity increases with increasing upper time limit, which is not a surprise, since a wider time window implies more events to be included. In contrast, the mean energy per photon is practically constant over the studied time range; the mean value is indicated by a dashed vertical line, which agrees well with previously published values $[4,5]$. This behavior is not really surprising either, since by widening the prompt time window the contributions from the additional $\gamma$ rays become smaller and smaller. This implies that the measured energy spectra for each of these time windows should have the same shape, which by unfolding of the response function must lead to emission spectra, which have the same shape, too. Hence, the number of events within a certain window of the time-of-flight distribution should be proportional to the average multiplicity obtained by means of a proper unfolding of the measured energy spectrum of the corresponding $\gamma$ rays. This is demonstrated by integrating over the TAC spectrum from a certain lower time limit to any upper time limit, which normalized to the actually determined multiplicities - results in the line in the left part of Fig. 4 , in perfect agreement with the experimental values. Finally, since $\bar{\epsilon}_{\gamma}$ is practically 

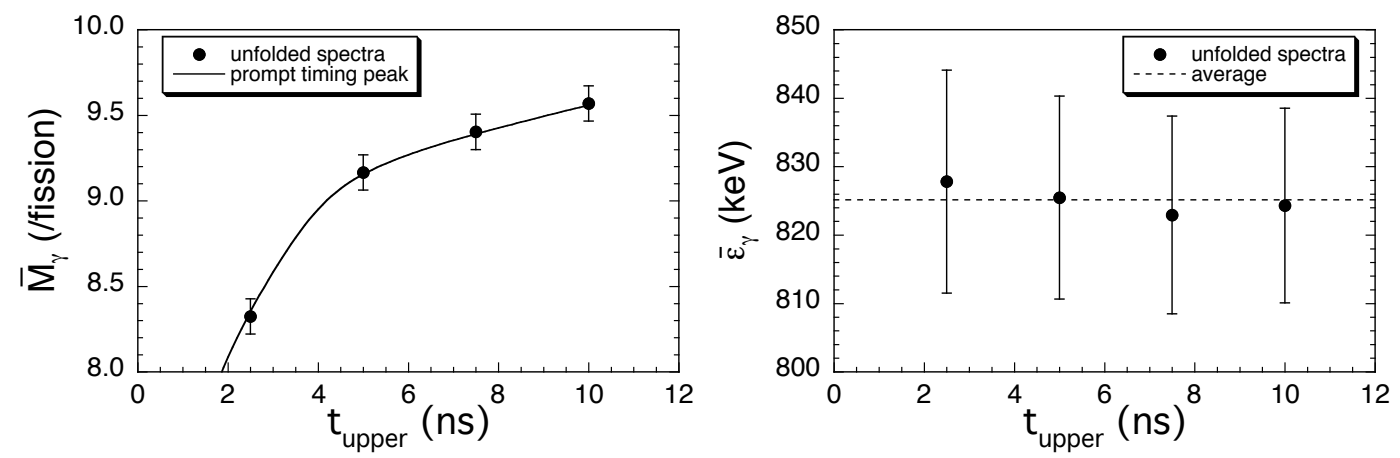

Figure 4. Average prompt fission $\gamma$-ray multiplicity $\bar{M}_{\gamma}$ and mean energy per photon $\bar{\epsilon}_{\gamma}$ from ${ }^{252} \mathrm{Cf}(\mathrm{sf})$, obtained for different prompt timing windows. The lower time limit is in all cases $-2.5 \mathrm{~ns}$, while the upper limits correspond to the regions in Fig. 3. See text for more details.

constant, we may conclude that the average total $\gamma$-ray energy released in fission, $\bar{E}_{\gamma, \text { tot }}=$ $\bar{M}_{\gamma} \times \bar{\epsilon}_{\gamma}$, exhibits the same behavior as $\bar{M}_{\gamma}$.

In order to generalize time-of-flight distributions of prompt $\gamma$ rays, we suggest to apply an approximation by a Lorentzian, as depicted in Fig. 3. This way it is possible to estimate multiplicities for any prompt timing window (albeit within reasonable limits) by simply integrating over an analytical mathematical function. With "reasonable limits" we refer here to the question, how late after fission a $\gamma$ ray may still be considered prompt. This question was discussed in Ref. [18] and will be addressed by us elsewhere. Anyway, since the width of the prompt peak is a measure for the experimental coincidence timing resolution, it varies usually from experiment to experiment depending on employed detectors and other electronics. Hence, by expressing all times in units of the observed timing resolution, i.e. the full width at half maximum (FWHM) of the prompt peak, one becomes independent from particular experimental conditions.

Figure 5 shows results of those integrations in form of a nomogram. A relative multiplicity is plotted as a function of the upper integration limit $t_{\text {upper }}$ and parametrized by the lower limit $\mathrm{t}_{\text {lower }}$. All times are given in units of the timing resolution $\Delta \mathrm{t}_{F W H M}$, in order to be applied to any experimental condition. The full drawn lines connect points of same value for $\mathrm{t}_{\text {lower }}$ and varying $\mathrm{t}_{\text {upper }}$, describing asymmetric prompt timing windows. Results for symmetric timing distributions, i.e. for $t_{\text {upper }}=-t_{\text {lower }}$, are depicted as dotted line. Here, a value of one for the relative multiplicity has been chosen for a symmetric window which is $5 \times \Delta t_{F W H M}$ wide. If a prompt fission $\gamma$-ray multiplicity has been determined for a certain timing window and a corresponding value for another experiment and/or timing window is to be inferred, the nomogram in Fig. 5 may be used as follows: for a performed multiplicity measurement with known timing resolution and prompt window as reference, the relative multiplicity is read depending on the limits of the chosen time window. Then the same is done for experimental conditions for which the result needs to be estimated. Finally, the new absolute multiplicity value is obtained by multiplying the reference value with the ratio of both relative multiplicities. As an example data from Ref. [11] is added in Fig. 5 as black dots. They were obtained for ${ }^{252} \mathrm{Cf}(\mathrm{sf})$ with a coincidence timing resolution of $\Delta \mathrm{t}_{F W H M}=1.25 \mathrm{~ns}$ and for different symmetric prompt windows. According to the convention chosen here, a relative multiplicity of one is associated with a total witdth of $6.25 \mathrm{~ns}$. Obviously, the data points follow very well the predicted dependence shown by the dotted line, thus indicating that the suggested 


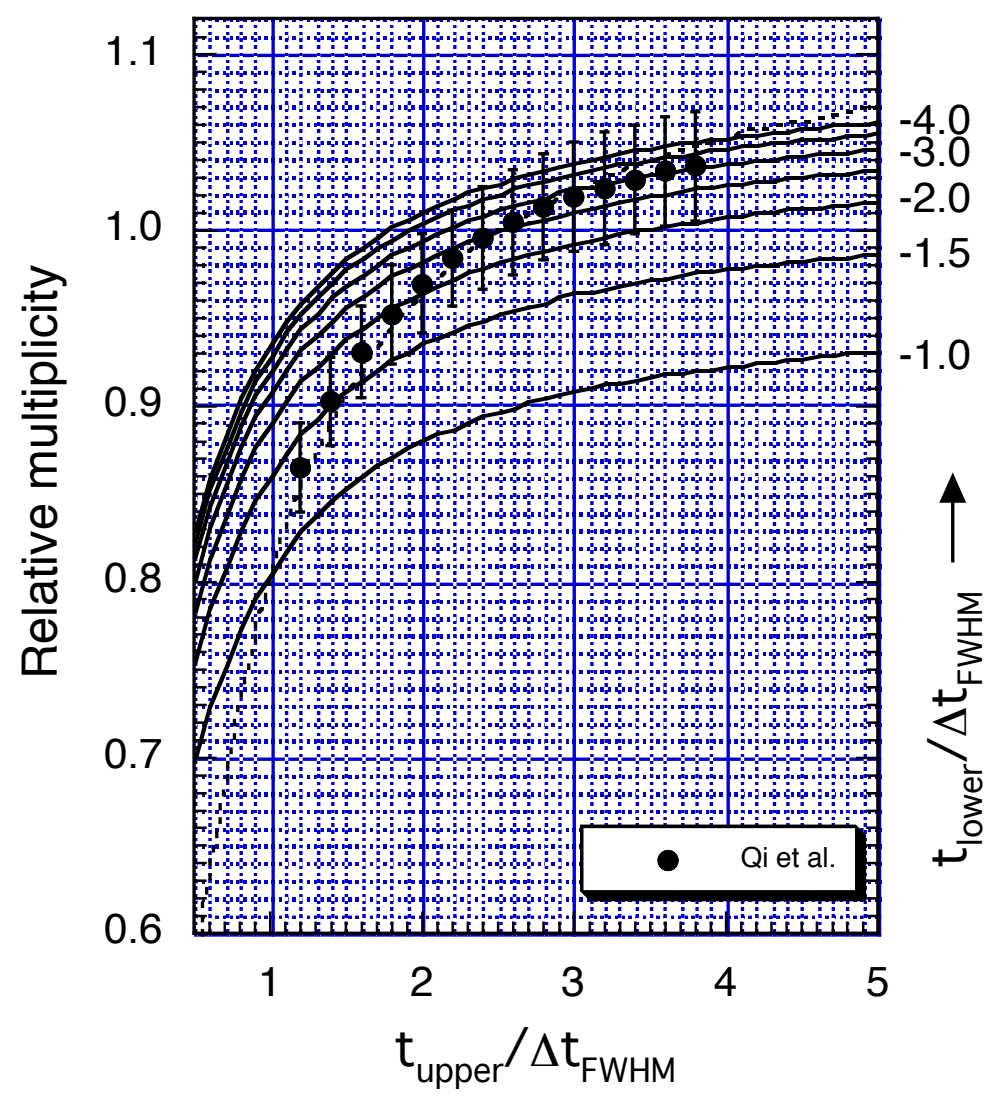

Figure 5. Nomogram to illustrate the calculated dependence of the (relative) prompt $\gamma$-ray multiplicity from the width of the chosen time window around the instant of fission. Upper and lower time limits are given in units of coincidence timing resolution $\triangle \mathrm{t}_{F W H M}$ (see text for details). Experimental data from Qi et al. [11] are shown for comparison.

procedure is indeed a useful tool to compare prompt fission $\gamma$-ray multiplicities from different experiments, i.e. to compare "apples and oranges".

\section{Discussion and outlook}

In the present work we have reported on two aspects of the measurement of fission fragment de-excitation by prompt $\gamma$-ray emission.

For the system $n+{ }^{235} \mathrm{U}$ we have presented the very first results for fast-neutron induced prompt fission $\gamma$-ray characteristics, which was achieved by combining results from a previously performed comparative measurement [15] and a recent study on $n+{ }^{238} U$ [11] at almost identical incident neutron energy. The deduced PFGS characteristics are in rather good agreement with a recent measurement with thermal neutrons [16] - within the uncertainties. Still, all values are somewhat lower for fast neutrons than for thermal ones, which is certainly opposite to our expectations [14], but this was already observed for the system $n+{ }^{239} \mathrm{Pu}[8,11]$. It has to be admitted that the statistics was quite low, but nevertheless, it is the published first PFGS results for fast neutrons on ${ }^{235} \mathrm{U}$. Obviously, a new measurement with better statistics is highly recommended. 
In detailed studies of the spontaneous fission of ${ }^{252} \mathrm{Cf}$ we investigated the dependence of PFGS characteristics from the width of the chosen prompt time window. Within the range \pm 10 ns we observed a with time increasing (but apparently saturating) average prompt $\gamma$-ray multiplicity - and consequently total $\gamma$-ray energy, since the average energy per photon appears to be constant. This behavior could be explained by integration over the prompt timing distribution within different limits. By expressing the time dependence in units of a respective experimental coincidence timing resolution, conclusions can be made independent from a particular experiment. Moreover, results from different measurements can be compared with each other, which has been illustrated graphically. In the next future, we will extend the investigated time scale by several orders of magnitude to address the questions of how late after the instant of fission emitted $\gamma$ rays may still be considered prompt and how experimental findings compare to theoretical predictions.

One of the authors (A. O.) acknowledges the support from the Extreme Light Infrastructure Nuclear Physics (ELI-NP) Phase II, a project co-financed by the Romanian Government and the European Union through the European Regional Development Fund - the Competitiveness Operational Programme (1/07.07.2016, COP, ID 1334), with which this work had been finalized.

\section{References}

[1] R. Vandenbosch and J.R. Huizenga, Nuclear Fission, Academic Press New York and London (1973)

[2] C. Wagemans (editor), The Nuclear Fission Process, CRC Press Boca Raton (1991)

[3] S. Oberstedt, R. Billnert, F.-J. Hambsch, M. Lebois, A. Oberstedt, and J.N. Wilson, Eur. Phys. J. A 51, 178 (2015)

[4] R. Billnert, F.-J. Hambsch, A. Oberstedt, and S. Oberstedt, Phys. Rev. C 87, 024601 (2013)

[5] A. Oberstedt, R. Billnert, F.-J. Hambsch, and S. Oberstedt, Phys. Rev. C 92, 014618 (2015)

[6] S. Oberstedt, A. Oberstedt, A. Gatera, A. Göök, F.-J. Hambsch, A. Moens, G. Sibbens, D. Vanleeuw, and M. Vidali, Phys. Rev. C 93, 054603 (2016)

[7] A. Oberstedt, T. Belgya, R. Billnert, R. Borcea, T. Bryś, W. Geerts, A. Göök, F.-J. Hambsch, Z. Kis, T. Martinez, S. Oberstedt, L. Szentmiklosi, K. Takács, and M. Vidali, Phys. Rev. C 87, 051602(R) (2013)

[8] A. Gatera, T. Belgya, A. Göök, F.-J. Hambsch, M. Lebois, A. Oberstedt, S. Oberstedt, L. Qi, L. Szentmiklósi, G. Sibbens, and M. Vidali, Phys. Rev. C 95, 064609 (2017)

[9] S. Oberstedt, R. Billnert, T. Belgya, T. Bryś, W. Geerts, C. Guerrero, F.-J. Hambsch, Z. Kis, A. Moens, A. Oberstedt, G. Sibbens, L. Szentmiklosi, D. Vanleeuw, and M. Vidali, Phys. Rev. C 90, 024618 (2014)

[10] M. Lebois, J.N. Wilson, P. Halipré, A. Oberstedt, S. Oberstedt, P. Marini, C. Schmitt, S. J. Rose, S. Siem, M. Fallot, A. Porta, and A.-A. Zakari, Phys. Rev. C 92, 034618 (2015)

[11] L. Qi, M. Lebois, J.N. Wilson, A. Chatillon, S. Courtin, G. Fruet, G. Georgiev, D. G. Jenkins, B. Laurent, L. Le Meur, A. Maj, P. Marini, I. Matea, L. Morris, V. Nanal, P. Napiorkowski, A. Oberstedt, S. Oberstedt, C. Schmitt, O. Serot, M. Stanoiu, and B. Wasilewska, Phys. Rev. C 98, 014612 (2018)

[12] J.-M. Laborie, R. Billnert, G. Bélier, A. Oberstedt, S. Oberstedt, and J. Taieb, Phys. Rev. C 98, 054604 (2018) 
[13] L. Qi, J.N. Wilson, M. Lebois, A. Al-Adili, A. Chatillon, D. Choudhury, A. Gatera, G. Georgiev, A. Göök, B. Laurent, A. Maj, I. Matea, A. Oberstedt, S. Oberstedt, S.J. Rose, C. Schmitt, B. Wasilewska and F. Zeiser, EPJ Web of Conf. 169, 00018 (2018)

[14] A. Oberstedt, R. Billnert, and S. Oberstedt, Phys. Rev. C 96, 034612 (2017)

[15] M. Lebois, J.N. Wilson, P. Halipré, B. Leniau, I. Matea, A. Oberstedt, S. Oberstedt, D. Verney, Nucl. Instr. Methods Phys. Res. A 735, 145 (2014)

[16] A. Gatera, PhD thesis, Ghent University (2019)

[17] A. Oberstedt, S. Oberstedt, R. Billnert, W. Geerts, F.-J. Hambsch, J. Karlsson, Nucl. Instr. Methods Phys. Res. A 668, 14 (2012)

[18] P. Talou, T. Kawano, I. Stetcu, J.P. Lestone, E. McKigney, and M. B. Chadwick, Phys. Rev. C 94, 064613 (2016) 